\title{
Correlation between maximum in-shoe plantar pressures and clubhead speed in amateur golfers
}

\begin{abstract}
Disagreements exist in the literature regarding the manner in which weight should be dynamically shared during the golf swing, both within-feet and between the back- and target-feet, to generate maximal clubhead speed. The purpose of this study was to determine whether preferential foot loading locations underly weight sharing by examining the correlation between clubhead speed and maximum plantar pressure (PP) distributions. Thirty-two amateur golfers with handicap indexes ranging from 2.7 to 25 performed ten driver swings on artificial turf following a warm-up. Plantar pressure distributions were recorded at $100 \mathrm{~Hz}$, and clubhead speed was recorded using a ball-tracking doppler-radar system. Maximum PPs were extracted from a $2 \mathrm{~s}$ window approximately centered on ball contact, and were regressed against clubhead speed. Significance was assessed over the entire foot surface using statistical parametric mapping (SPM), a spatially continuous technique. SPM revealed, at high anatomical resolution, significant positive correlations between clubhead speed and PPs in the lateral target-foot $(p<0.05)$. This suggests that not only weight-transfer but also weight-transfer location may be an important determinant of clubhead speed in amateur golfers.
\end{abstract}

Keywords: foot biomechanics, golf swing, pressure distribution, statistical parametric mapping 


\section{Introduction}

Forces acting at the foot-ground interface during the golf swing function partially to drive upperbody motion. It is therefore reasonable to assume that maximizing clubhead speed, a variable highly correlated with golf skill (Fradkin, Sherman, \& Finch, 2004), requires stereotypical and appropriately controlled foot forces. These forces can be easily measured and have been widely used for golf swing assessment (Richards, Farrell, Kent, \& Kraft, 1985; Ball \& Best, 2012), and it has been postulated that force sharing between the 'target' and 'back' feet (Fig.1), as manifested in parameters such as the center of pressure (COP), can differentiate amongst golfing skill levels (Richards et al., 1985; Koenig, Tamres, \& Mann, 1994) and thus, indirectly, clubhead speeds.

A variety of evidence from the literature supports this claim. For example, it has been independently verified that that highly skilled golfers exhibit greater $\mathrm{COP}_{y}$ excursion (i.e. in the target-direction) than less-skilled golfers (Richards et al., 1985; Ball \& Best, 2007). Other re-

sults clarify that that this increased excursion results primarily from increased target-foot loading and decreased back-foot loading $40 \mathrm{~ms}$ prior to ball contact (Chu, Sell, \& Lephart, 2010; Okuda, Gribble, \& Armstrong, 2010).

However, the manner in which golfers dynamically distribute forces across the back- and targetfeet is less clear. One could, for example, start with a loaded back-foot heel and then finish with loaded target-foot toes, and this would achieve the same COPy excursion as starting with the toes and finishing with the heel. It could be argued that these two extremes differentially constrain swing path and ball contact possibilities.

There have only been a few studies to-date that help to distinguish amongst different force sharing solutions. One study suggests that the target foot heel should be loaded through ball contact (Richards et al., 1985), and this is supported anecdotally through observations that "target foot golfers", who have a more posterior COPx through ball impact, have lower average handicaps (Ball \& Best, 2011). It is also indirectly supported by observations that single-digit handicappers load the posterior and lateral target-foot (Murakami \& Mochimaru, 2010). Somewhat contradictory to these findings are observations that both heel and hallux forces, but not lateral forces, are associated with more skilled golfers (Kawashima, Meshizuka, \& Takeshita, 1998). 
One possible explanation for these discrepancies is the fact that all studies to-date fail to treat foot force distributions as spatially continuous variables. COP studies consider only single points (Ball \& Best, 2011), so are unable to determine whether both toe and heel loads, for example, vary systematically with clubhead speeds. Discrete sensor studies, which examine only a small number of foot locations (Kawashima et al., 1998) cannot describe all forces acting across the foot surface. Indeed, of the only two studies that employed spatially continuous measurements of the foot surface, one did not test different clubhead speeds or skill levels (Murakami \& Mochimaru, 2010) and the other resolved the spatially continuous data to COP point-trajectories (Healy, 2009).

A second limitation of previous studies is that neither clubhead speed nor skill level was treated as a continuous variable. This is problematic, not only because terms like 'high skill' and 'low handicap' are imprecisely defined, making it difficult to resolve inter-study differences, but more importantly because a categorical comparison of clubhead speed (or skill-level) groups is inherently a weaker statistical design than (continuous) regression analysis.

The purpose of this study was to examine the correlation between spatially continuous foot force distributions and clubhead speed in amateur golfers in attempts to resolve discrepancies in published descriptions of foot-loading solutions in high- vs. low-skill golfers. To this end, the presently tested null hypothesis was: maximum in-shoe plantar pressure distributions occurring during the golf swing do not correlate with clubhead speed.

\section{Methods}

\section{$2.1 \quad$ Experiment}

Ethical approval for this experiment was obtained from Shinshu University, and informed consent was obtained from all subjects prior to their participation. In total 32 right-handed male amateur golfers (age: $45.2 \pm 13.1$ years, height: $176.2 \pm 6.0 \mathrm{~cm}$, mass: $76.4 \pm 7.7 \mathrm{~kg}$ ) volunteered to participate in this experiment. Their self-reported handicap indexes ranged from 2.7 to 25 (average: $15.8 \pm 6.2)$.

Each participant stood on an artificial turf mat at a driving range, and hit ten driver shots 
from a rubber tee. All participants warmed up with 10-20 practice swings, then 10 consecutive swings were recorded. Clubhead speed was recorded with a Flightscope X2 doppler radar launch monitor (EDH Ltd., Stellenbosch, South Africa), a system with a manufacturer-specified accuracy of approximately 99\%, achieved through constant during-flight ball monitoring. In-shoe plantar foot pressures were recorded at $100 \mathrm{~Hz}$ using the Pedar X insole system (Novel GmbH, Munich, Germany), a system with 99 sensors in each insole, and with an accuracy of approximately $98 \%$ (Hsiao, Guan, \& Weatherly, 2002). To minimize shoe design effects all subjects were fitted with the same golf shoe model (Lunarlon Control, Nike Inc.) prior to the experiment.

\subsection{Data processing}

Analyses focussed on a $2 \mathrm{~s}$ window in the vicinity of ball contact. For each trial, the instants of maximum back- and target-foot ground reaction forces (GRF) were identified, and the instant halfway between them was used as the center of the $2 \mathrm{~s}$ window-of-interest. Pilot investigations with a time-synchronized web-camera $(25 \mathrm{~Hz})$ verified that ball impact always occurred close to the center of this $2 \mathrm{~s}$ window. Maximum plantar pressures (PP) were extracted from each sensor over the $2 \mathrm{~s}$ window-of-interest, yielding one maximum PP distribution per trial.

\section{$2.3 \quad$ Statistics}

The relation between maximum PP and clubhead speed was modeled as a random-effects linear regression:

$$
\bar{y}=m_{i} \overline{x_{i}}+y_{0 i}
$$

where $\bar{y}$ is the within-subject average clubhead speed, $\overline{x_{i}}$ is the is the within-subject average maximum PP observed at the $i$ th sensor, and $m_{i}$ and $y_{0 i}$ are the $i$ th sensor's slope and intercept, respectfully. While Eqn.1 neglects within-subject variability, this model is equivalent to a full model incorporating both between- and within-subject variability when the model residuals are normally distributed (Friston, Ashburner, Kiebel, Nichols, \& Penny, 2007). In other words, each subject's mean clubhead speed and mean PP distribution were regarded as fixed to that subject, 
but the particular subjects studies were regarded as randomly drawn from the population of all amateur golfers.

Fitting Eqn.1 to the data in a least-squares sense yielded one correlation coefficient $(r)$ per sensor and thus one $r$ distribution per subject. This $r$ distribution was converted to a $t$ statistic distribution using the identity:

$$
t=\sqrt{(n-2) /\left(1-r^{2}\right)}
$$

where $n$ is the number of subjects $(n=32)$, and where $t$ has the Student's t distribution with $(n-2)$ degrees of freedom.

Statistical inference was conducted using statistical parametric mapping (SPM) (Adler \& Taylor, 2007; Friston et al., 2007), a methodology which generalizes statistical inference from the typical zero-D case (e.g. maximum vertical force) to multi-dimensional continuum data (2D scalar PP distribution in this study). For a zero-D regression test, one sets $\alpha$, then computes the critical $r$ (and $t$ ) value above which normally distributed random data would be expected to traverse in only $\alpha \%$ of repeated experiments. Identically, in a $2 \mathrm{D}$ regression test, one sets $\alpha$ then computes the critical $r$ (and $t$ ) above which normally distributed smooth 2D random data would be expected to traverse in only $\alpha \%$ of repeated experiments. In both cases the presence of suprathreshold $r$ (or t) values provide sufficient evidence to reject the null hypothesis of no correlation. This inference procedure has been validated elsewhere for PP data (Pataky, 2008).

\section{Results}

The average PP distribution was asymmetric (Fig.2), with the highest target-foot pressures acting on the lateral forefoot, heel and hallux, and with the highest back-foot pressures contrastingly acting on the medial forefoot and hallux. Different subjects produced qualitatively different PP

distributions (Fig.3); whereas subjects with the highest clubhead speeds all tended to produce high lateral target-foot pressures (Fig.3, bottom panels), subjects with lower clubhead speeds tended to not load the lateral target-foot to the same extent (Fig.3, top panels), and also to have more 
variable PP distributions. Although heavier subjects tended to produce higher clubhead speeds, post hoc linear regression between clubhead speed and body mass was moderately weak $(r=0.315)$ and failed to reach significance $(p=0.079)$.

For an example lateral target-foot sensor (Fig.2) a moderately strong positive linear correlation was observed between pressure and clubhead speed (Fig.4). In whole-foot analysis pressures were positively correlated with clubhead speed in the distal back-foot and over most of the target-foot (Fig.5a). Weak negative correlations were observed on the posterior back-foot and on the posterior heel and medial forefoot of the target-foot. Positive correlations reached significance at $\alpha=0.05$, but only at the lateral target-foot and the very distal back-foot (Fig.5b). The present null hypothesis was therefore rejected. Negative correlations failed to reach significance at $\alpha=0.05$. Post hoc analyses testing the linear relation between within-subject standard deviation distributions and clubhead speed failed to reach significance $(p>0.05)$.

\section{Discussion}

\subsection{Present vs. previous results}

The present positive correlations between target-foot pressures and clubhead speed (Fig.5a) support the conventional wisdom that weight-transfer to the target-foot is important for generating clubhead speed (Hogan \& Wind, 1957), and they also agree with scientific findings of higher targetfoot forces in skilled vs. unskilled golfers (Richards et al., 1985; Ball \& Best, 2007; Okuda et al., 2010). The present results additionally suggest that loading the lateral target-forefoot may be particularly important for generating clubhead speed (Fig.5b). While previous results imply increased lateral target-foot loading in categorical analyses of skilled vs. unskilled golfers (Ball \& Best, 2007; Kawashima et al., 1998), the present results are the first to demonstrate significant correlation between the PP distribution and actual clubhead speed.

The main novelty of the present results is thus that they indicate that target-foot loading location may be as important as target-foot loading itself for generating clubhead speed. In particular, neither ground reaction force nor COP can reveal which parts of the foot should be loaded. 
Although foot loading locations can be inferred from COP data, COP data are anatomically ambiguous because one COP location can be produced by many PP distributions.

It has been previously shown that mechanical intervention in the form of passive heel-lifts can increase within-subject driving distance (Rambarran \& Kendall, 2001). If these heel-lifts redistribute pressures anteriorly and/or laterally, then Rambarran and Kendall's results would be consistent with those in Fig.5a.

The present results nevertheless contradict reports of higher target-foot hallux loads in skilled golfers vs. unskilled golfers (Kawashima et al., 1998). Similarly, they contradict reports that medial target-foot pressures are associated with skilled golfers' longest drives (Wallace, Grimshaw, \& Ashford, 1994). However, since these previous reports focussed on low-handicap golfers (handicap $<10$ ), and since handicap is negatively correlated with clubhead speed (Fradkin et al., 2004), it is possible that the present result reflects a trend in a broader range of skill levels, and thus that the results of all studies would converge for a constant skill-level range.

Since significance was presently limited to the anterior half of the foot, the present results also contradict findings that $\mathrm{COP}_{x}$ positions do not differ between $<3$ and 10-18 handicap golfers (Healy, 2009). However, the cited study employed barefoot testing, which likely involved both reduced foot stability and reduced foot-ground friction with respect to shod testing.

Although the present results suggest that loading arbitrary target-foot locations, especially the medial midfoot or posterior heel, may be counterproductive (Fig.5a), this trend must be interpreted cautiously because it may be attributable to morphological rather than functional subject differences. As may be inferred from Fig.3, it is possible that subjects with low clubhead speeds also had low medial arches. Arch-matched participants should therefore be tested to clarify this trend.

\subsection{Statistical parametric mapping (SPM)}

Unlike previous PP distribution studies which investigated a small handful of discrete foot regions, effects were presently assessed over the entire foot surface using statistical parametric mapping (SPM) (Friston et al., 2007). The SPM method offers greater anatomical objectivity because it does not artificially discretize the foot; it instead treats the foot as a continuous surface 
and assesses PP effects at high spatial resolution. This is important because the spatial frequencies of PP data are often higher than can be accurately captured with (low-resolution) discrete foot regions (Pataky, 2008). The main advantage of SPM is thus anatomical detail. It provides a clear anatomical context to the statistical data (Fig.5), making it easy to understand whole-foot effects at a quick glance. Tabulated pressure data, bar graphs, and scatter plots at discrete locations (Fig.4) are more difficult to interpret because one has to mentally map these data back to their original locations in order to perceive anatomical context. This problem is compounded when multiple anatomical regions must be considered simultaneously.

The whole-foot SPM approach (Fig.5a) may be especially useful as a coaching tool because 'hot' and 'cool' foot areas highlight the anatomy upon which coaches and athletes should focus. Even when other data like COP produce very convincing results (Ball \& Best, 2011), they may be unintuitive for instructional purposes: "move your COP laterally" is less clear than "transfer your weight to your lateral target-foot". Furthermore, since large target-direction COP movement can result from opposite-direction center-of-mass acceleration, the relation between COP movement and 'weight transfer' is mechanically tenuous.

\subsection{Limitations}

Present analyses were limited to between-subject effects. In pilot studies and in post hoc analyses of the present dataset (results not presently reported) insufficient evidence existed to reject the null hypothesis of no within-subject correlation between PP distribution and clubhead speed. This is somewhat unsurprising considering that golf-swing performance stems from a large variety of biomechanical determinants (Ball \& Best, 2012). Nevertheless, a lack of within-participant correlation does not preclude the possibility that ideal swing mechanics involve lateral target-foot loading. Additionally, since golf-swing performance determinants vary substantially from participant-toparticipant (Ball \& Best, 2012), it is possible that individually-tailored regression analyses would elucidate within-participant effects. Since within-subject effects are also relevant to the present null hypothesis, this paper's conclusions are therefore limited to only between-subject effects.

The present study did not consider temporal events (e.g. start of the downswing, ball impact). 
Instantaneous PP distribution analysis was presently avoided, not only because the present lowfrequency web-camera $(25 \mathrm{~Hz})$ made it difficult to precisely identify swing events like ball contact, but also because this was the first study of continuous PP distributions. Since temporal events at the foot-ground interface are important indicators of skill level (Richards et al., 1985; Chu et al., 2010) it is likely that instantaneous PP distributions would further elucidate clubhead speedrelevant trends.

\subsection{Summary}

A significant positive correlation between lateral target-foot pressures and clubhead speed appears to exist in amateur golfers, suggesting that target-foot loading location may be as important as target-foot loading itself for maximizing clubhead speed. This study was the first to assess pressure continuously across the entire plantar surface, yielding an easy-to-understand yet anatomicallydetailed summary of foot mechanics. Because of its anatomical clarity this whole-foot approach appears to represent a useful focus for further investigations of golf swing mechanics.

\section{References}

Adler, R. J., \& Taylor, J. E. (2007). Random fields and geometry. New York: Springer.

Ball, K. A., \& Best, R. J. (2007). Different centre of pressure patterns within the golf stroke II: group-based analysis. Journal of Sports Sciences, 25(7), 771-779.

Ball, K. A., \& Best, R. J. (2011). Golf styles and centre of pressure patterns when using different golf clubs. Journal of Sports Sciences, 29(6), 587-590.

Ball, K. A., \& Best, R. J. (2012). Centre of pressure patterns in the golf swing: individual-based analysis. Sports Biomechanics, 11(2), 175-189.

Chu, Y., Sell, T. C., \& Lephart, S. M. (2010). The relationship between biomechanical variables and driving performance during the golf swing. Journal of Sports Sciences, 28(11), 1251-1259.

Fradkin, A. J., Sherman, C. A., \& Finch, C. F. (2004). How well does club head speed correlate with golf handicaps? Journal of Science and Medicine in Sport, 7(4), 465-472. 
Friston, K. J., Ashburner, J. T., Kiebel, S. J., Nichols, T. E., \& Penny, W. D. (2007). Statistical parametric mapping: The analysis of functional brain images. London: Elsevier.

Healy, A. (2009). Identification of the biomechanical performance determining factors of the 5 iron golf swing when hitting for maximum distance. (Mater's Thesis, Dublin City University, Ireland). Retrieved from http://doras.dcu.ie/14864/

Hogan, B., \& Wind, H. W. (1957). The modern fundamentals of golf. London: Kaye \& Ward.

Hsiao, H., Guan, J., \& Weatherly, M. (2002). Accuracy and precision of two in-shoe pressure measurement systems. Ergonomics, 8, 537-555.

Kawashima, K., Meshizuka, T., \& Takeshita, S. (1998). A kinematic analysis of foot force exerted on the soles during the golf swing among skilled and unskilled golfers. In Science and Golf III: Proceedings of the World Scientific Congress of Golf (pp. 40-45). London: Taylor \& Francis.

Koenig, G. M., Tamres, M., \& Mann, R. W. (1994). The biomechanics of the shoeground interaction in golf. In A. J. Cochran \& M. R. Farrally (Eds.), Science and Golf II: Proceedings of the World Scientific Congress of Golf (pp. 40-45). London: Taylor \& Francis.

Murakami, E., \& Mochimaru, E. (2010). Foot pressure, ground reaction force and 3d motion analysis of golf swing applied to spikeless golf shoe development. In Proceedings of the 6th World Congress of Biomechanics. New York: Springer.

Okuda, I., Gribble, P., \& Armstrong, C. (2010). Trunk rotation and weight transfer patterns between skilled and low skilled golfers. Journal of Sports Science and Medicine, 9, 127-133.

Pataky, T. C. (2008). Assessing the significance of pedobarographic signals using random field theory. Journal of Biomechanics, 41(11), 2464-2473.

Rambarran, K. K., \& Kendall, M. (2001). Plantar center of pressure and its effect on golf swing distance and accuracy. In Proceedings of the 5th symposium on footwear biomechanics. Retrieved from http://www.footwearbiomechanics.org/?q=node/35

Richards, J., Farrell, M., Kent, J., \& Kraft, R. (1985). Weight transfer patterns during the golf swing. Research Quarterly in Exercise and Sport, 56, 361-365.

Wallace, E. S., Grimshaw, P. N., \& Ashford, R. L. (1994). Discrete pressure profiles of the 
feet and weight transfer patterns during the golf swing. In A. J. Cochran \& M. R. Farrally (Eds.), Science and Golf II: Proceedings of the World Scientific Congress of Golf (pp. 25-32). London: Taylor \& Francis. 


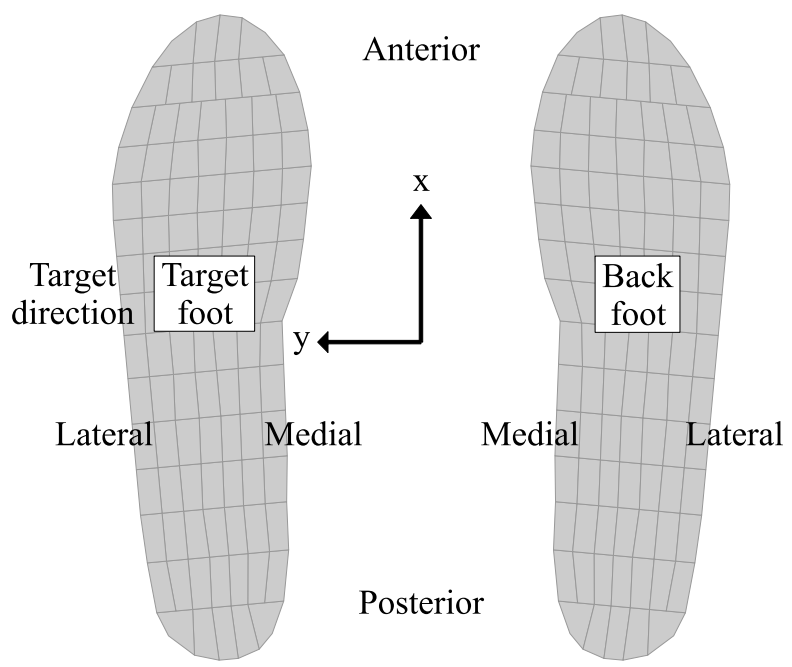

Figure 1: Terminology and coordinate system. 


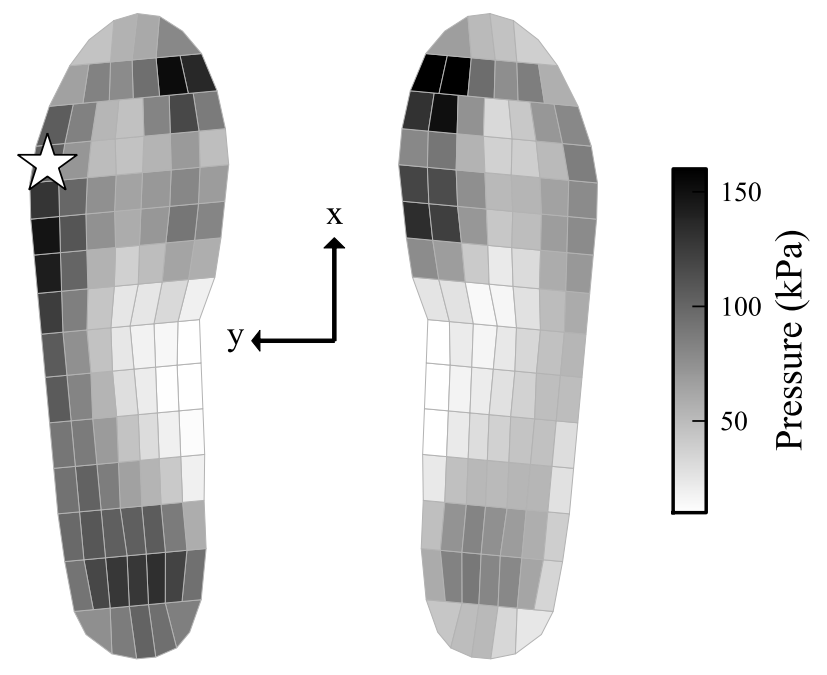

Figure 2: Maximum pressure distribution, averaged across subjects. The star symbol indicates the location for single-sensor analysis (Fig.4). 


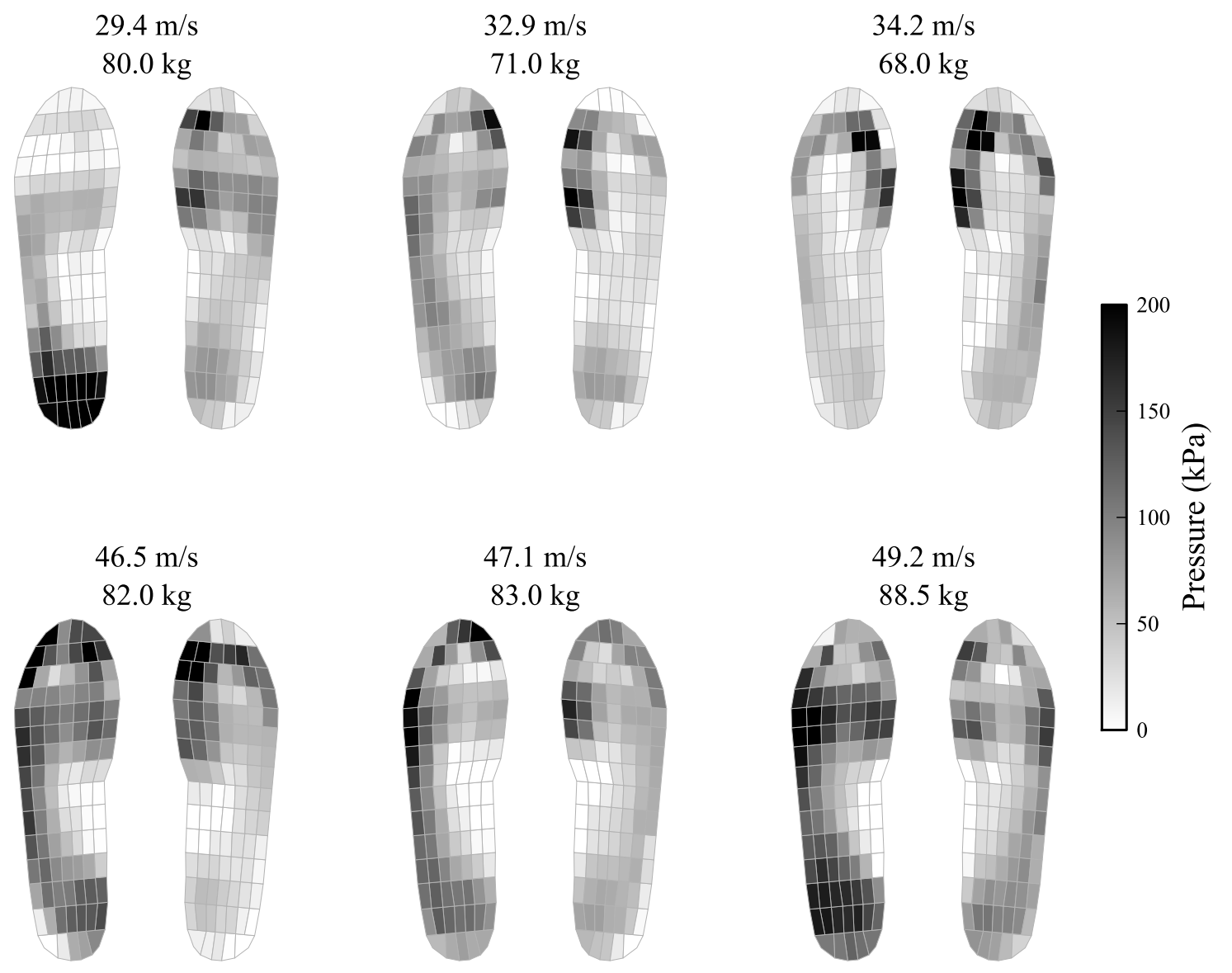

Figure 3: Maximum pressure distributions, averaged within-subjects. Top panel: the three subjects with the slowest clubhead speeds. Bottom panels: fastest clubhead speeds. Subjects' average clubhead speeds $(\mathrm{m} / \mathrm{s})$ and body mass $(\mathrm{kg})$ are indicated. 


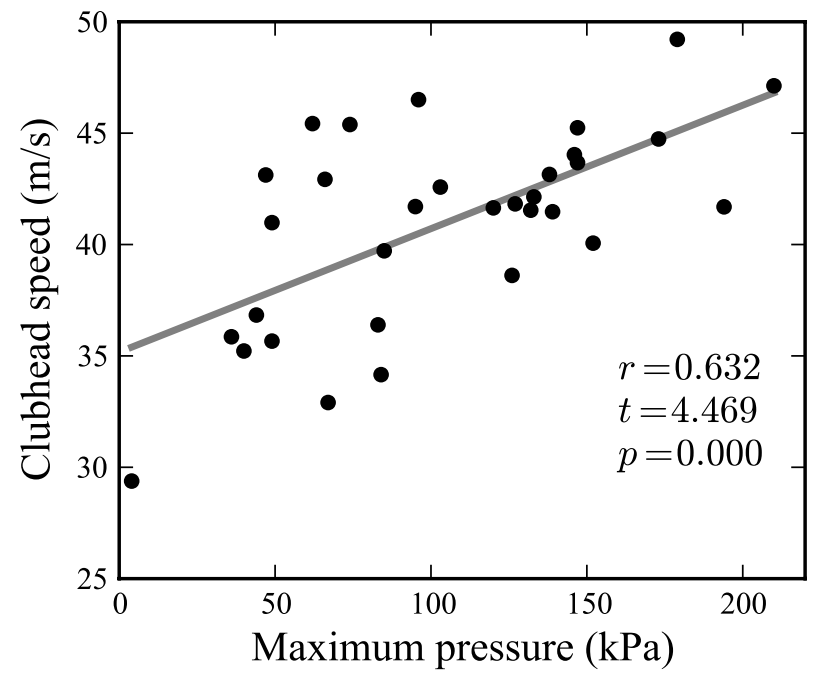

Figure 4. Linear regression results, single sensor. Data are from one sensor (location indicated in Fig.2), one point per subject representing the within-subject average across ten trials. 
(a) Raw $t$ distribution

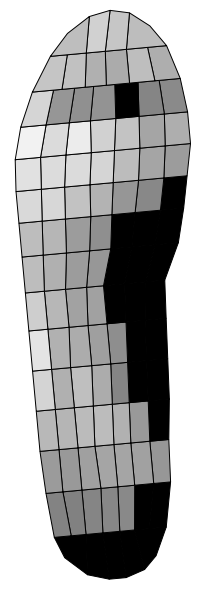

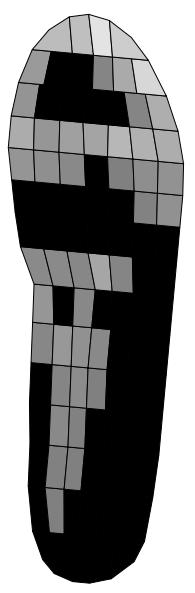

(b) Statistical inference

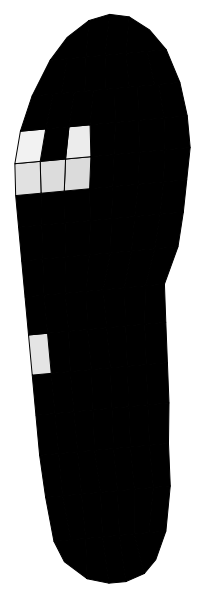

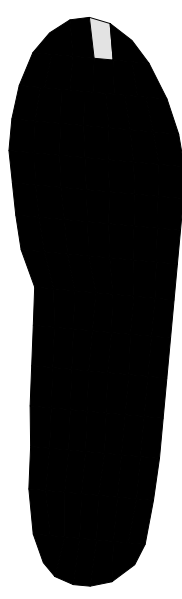

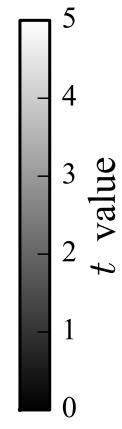

$\underline{\text { Figure 5. }}$. Linear regression results, whole foot. (a) Distribution of the $t$ statistic for all sensors; $\overline{\text { larger } t}$ values imply stronger positive correlation. (b) Statistical inference $(\alpha=0.05$; critical value: $|t|=3.58$ ). 